\title{
Contact Pressures and Cracks Identification by using the Dirichlet-to- Neumann Solver in Elasticity
}

\section{Mohamed Larbi Kadri ${ }^{\star}$}

Ecole Nationale d'Ingénieurs de Tunis (ENIT), Lamsin, Campus Universitaire, B.P. 37, 1002 Tunis, Tunisia

\begin{abstract}
In this work we present a numerical data completion method based on the Dirichlet-to-Neumann algorithm, by working in a linear elasticity framework. We begin by recasting the problem in terms of the Steklov-Poincare operator which is commonly used in domain decomposition. Then we present the Dirichlet-to-Neumann algorithm and state the equivalence between both formulations. The proposed method is applied to identify a contact pressures distribution and interfacial cracks.
\end{abstract}

Keywords: Inverse problem; Data completion; Cauchy problem; Identification; Dirichlet-to-Neumann algorithm

\section{Introduction}

Physical phenomena are often governed by partial differential equations, which need an essential set of data to solve them. In linear elasticity, these data are: the geometry of the solid, the mechanical properties of the materials and the boundary conditions. However, in many industrial applications, some of these data are unknown and have to be identified. This leads to an inverse problem whose resolution requires over-specified measured data. In this paper we focus on a problem of boundary condition identification in linear elasticity. In this case, data measured on part of the easily accessible border are often available. However, contrary to the direct problem, two kinds of boundary conditions (e.g. displacements and tractions) are imposed on the same part of the boundary while no information exists on the remaining part of it. Hence, data completion consists in reconstructing the boundary conditions for the whole boundary of a domain by using the partially overspecified measurements. This is the well-known Cauchy problem, which is ill-posed.

The ill-posedness of inverse problems may concern the existence and/or the uniqueness of the solution, but their most critical feature is their instability: the solution, whenever a problem has one, is not continuous with respect to the data, i. e. small measurement errors in the data may dramatically amplify the errors in the solution. This is illposedness in the Hadamard sense [1]. The Cauchy problem pertains to this kind of inverse problem. Therefore suitable regularizing algorithms that are exempt from this ill-posedness phenomenon, are required in order to solve the inverse problem correctly.

The Cauchy problem in linear elasticity was first studied by Yeih et al. [2]. In this paper, the existence and uniqueness of the solution are analyzed as well as the continuity of the solution with respect to the data. Others authors have proposed an alternative regularization procedure, namely the indirect fictitious boundary method, which is based on the simple or the double layer potential theory. The numerical implementation of the aforementioned method has been carried out by Koya et al. [3] who used the BEM and the Nystrom method for discretizing the integrals involved. Marin et al. [4] have determined the approximate solutions of the Cauchy problem in linear elasticity using an alternating iterative BEM that reduces the problem to solving a sequence of well-posed boundary value problems [5]. In Marin and Lesnic have used singular value decomposition to solve the same problem numerically. A related inverse problem which allows for interior displacement measurements and inter-facial crack has been investigated by Huang and Shih [6]. In Weikel et al. [7] have proposed an alternating iterative algorithm in order to reconstruct an internal planar crack laying on an a priori known internal surface inside a threedimensional elastic body from over determined elastostatic boundary data on the outer surface. Furthermore, Koslov and his co-authors adapted the iterative Dirichlet-to-Neumann method to approximate the solution of the Cauchy-Poisson problem, governed by the Laplace equation, and they provide proof of its convergence and its regularizing properties [8]. In [9] the iterative Dirichlet-to-Neumann method was applied to recover the missing boundary data for the Cauchy Helmholtz problem. More recently kadri et al. [10] have used the SteklovPoincaré approach relying on domain decomposition for the identification of internal planar cracks inside a three-dimensional using elastostatic measurements.

In this work, the iterative Dirichlet-to-Neumann method is applied to the linear elastic data completion problem. In section 2, the Cauchy problem is presented in the context of linear elasticity. In section 3 this problem is recast in condensed form that we will refer to as the Cauchy-Steklov-Poincaré problem, which leads to the Cauchy-SteklovPoincaré equation acting on the boundary of the unknowns. In section 4 we present the Dirichlet-to-Neumann algorithm and we show that it can be interpreted as a preconditioned Richardson procedure for the Cauchy-Steklov-Poincaré equation. The numerical procedure and the results obtained by FEM discretization of the problem are presented in section 5. The method is used to solve two applications borrowed from engineering mechanics: the identification of contact pressures and coating defect in a double layered composite domain.

\section{The Cauchy Problem in Linear Elasticity}

Let denote a bounded domain in $\mathbb{R}^{2}$ or $\mathbb{R}^{3}$ with regular boundary $\Gamma=\partial \Omega$. The whole domain is assumed to be filled with a homogeneous linear elastic isotropic medium. It is assumed th at $\Gamma$ is splitted into two

${ }^{*}$ Corresponding author: Mohamed Larbi Kadri, Ecole Nationale d'Ingénieurs de Tunis (ENIT), Lamsin, Campus Universitaire, B.P. 37, 1002 Tunis, Tunisia, E-mail: medlarbi.kadri@lamsin.rnu

Received September 01, 2014; Accepted January 20, 2015; Published January 28,2015

Citation: Kadri ML (2015) Contact Pressures and Cracks Identification by using the Dirichlet-to-Neumann Solver in Elasticity. J Appl Mech Eng 4: 152. doi:10.4172/2168-9873.1000152

Copyright: $\odot 2015$ Kadri ML. This is an open-access article distributed under the terms of the Creative Commons Attribution License, which permits unrestricted use, distribution, and reproduction in any medium, provided the original author and source are credited. 
open subsets $\Gamma_{c}$, and $\Gamma_{i}, \Gamma=\Gamma_{c} U \Gamma_{i}$, where $\Gamma_{c}, \Gamma_{i}, \Gamma_{i}=\phi$ and $\Gamma_{c} \cap \Gamma_{i}=\varnothing$. In what follows, $\mathrm{u}(\mathrm{X})$ denotes the displacements field on $\Omega$.

The local equilibrium equation is given by

$-\operatorname{div} \sigma(\mathbf{u}(\mathrm{x}))=\mathbf{f} \quad \mathrm{x} \in \Omega$,

where $\boldsymbol{\sigma}$ is the stress tensor and $\mathbf{f}$ the volume forces. The strain tensor $\varepsilon$ is given by

$$
\varepsilon(\mathbf{u}(\mathrm{x}))=\frac{1}{2}(\nabla \mathbf{u}(\mathrm{x})+\nabla \mathbf{u}(\mathrm{x}))
$$

These tensors are related by the Hooke's constitutive law, which is

$$
\sigma(\mathbf{u}(\mathrm{x}))=2 \mu \varepsilon(\mathbf{u}(\mathrm{x}))+\lambda \operatorname{tr} \varepsilon(\mathbf{u}(\mathrm{x})) \mathrm{I}=\mu(\nabla \mathbf{u}(\mathrm{x})+\nabla \mathbf{u}(\mathrm{x}))+\lambda \operatorname{div} \mathbf{u}(\mathrm{x})
$$

where $\lambda$ and $\mu$ are the Lamé constants of the material and $\mathrm{I}$ is the identity tensor.

Let $\mathrm{n}(\mathrm{x})$ be the outward normal vector at $\Gamma$ and $\mathrm{t}(\mathrm{x})$ be the traction vector at a point $\mathrm{x} \in \Gamma$ defined by

$$
\mathbf{t}(\mathrm{x})=\sigma(\mathbf{u}(\mathrm{x})) \sigma \mathbf{n}(\mathrm{x}) \mathrm{x} \in \Gamma
$$

In the well-posed direct problem formulation, the knowledge of the displacement on a part of the boundary and traction vectors on another part of the boundary enables us to determine the displacement vector in domain $\boldsymbol{\Omega}$. Then, the strain tensor $\boldsymbol{\varepsilon}$ can be calculated from kinematic relation (2) and the stress tensor is determined by constitutive law (3).

If a part of the boundary $\Gamma_{i}$ is inaccessible and if it is possible to measure both the displacement and traction vectors on the remaining part of boundary $\Gamma_{c}$, this leads to the mathematical formulation of a direct problem expressed as follows: $\begin{cases}-\operatorname{div} \sigma(\mathbf{u}(\mathrm{x}))=f & \text { in } \Omega \\ u(\mathrm{x})=\tilde{v}(\mathrm{x}) & \text { on } \Gamma_{\mathrm{c}} \\ t(\mathrm{x})=\tilde{\mathbf{t}}(\mathrm{x}) & \text { on } \Gamma_{\mathrm{c}}\end{cases}$

Where $\tilde{\mathbf{u}}$ and $\tilde{\mathbf{t}}$ are prescribed vector valued functions. This problem is ill-posed because of the formulation of its boundary conditions (5). It can be seen that boundary $\Gamma_{c}$ and the traction tis overs pecified by prescribing both the displacement $v_{\Gamma_{\mathrm{c}}}=\tilde{v}$ and the tractions $\mathbf{t}_{\Gamma_{\Gamma}}=\tilde{\mathbf{t}}$ vectors, while boundary $\Gamma_{i}$ underspecified since both the displacement $\mathbf{u}_{\mid \Gamma_{i}}=\overline{\mathbf{u}}$ and the traction $\mathbf{t}_{\Gamma_{i}}=\overline{\mathbf{t}}$ are unknown and have to be determined. Then, this problem can be stated as follows: find $(\overline{\mathbf{u}}, \overline{\mathbf{t}})$ that a displacement field $\mathrm{u}(\mathrm{x})$ exists that satisfies:

$$
\left\{\begin{array}{lc}
-\operatorname{div} \sigma(\mathbf{u}(\mathrm{x}))=\mathbf{f} & \text { on } \Omega \\
\mathbf{u}(\mathrm{x})=\tilde{\mathbf{u}}(\mathrm{x}) & \text { in } \Gamma_{\mathrm{c}} \\
\sigma(\mathbf{u}(\mathrm{x})) \mathrm{n}=\tilde{\mathbf{t}}(\mathrm{x}) & \text { in } \Gamma_{\mathrm{c}} \\
\mathbf{u}(\mathrm{x})=\overline{\mathbf{u}}(\mathrm{x}) & \text { in } \Gamma_{\mathrm{i}} \\
\sigma(\mathbf{u}(\mathrm{x})) \mathbf{n}=\overline{\mathbf{t}}(\mathrm{x}) & \text { in } \Gamma_{\mathrm{i}}
\end{array}\right.
$$

This problem, known as the Cauchy problem, is ill-posed in the sense that the dependence of $\mathrm{u}(\mathrm{x})$, and consequently of $(\overline{\mathbf{u}}, \overline{\mathbf{t}})$, on the data $(\tilde{\mathbf{u}}, \tilde{\mathbf{t}})$ is not continuous. Although the problem may have a unique solution, it is well-known that this solution is unstable with respect to small parturbation in the data on $\Gamma_{c}$. In this paper we propose to recover the lacking data by using the Dirichlet-to-Neumann algorithm introduced by Kozlov et al. in the steady state thermal case [8]. However, let us first introduce an operator acting on the boundary where data are unknown: the Steklov-Poincaré operator which is very familiar in domain decomposition and recently introduced for the Cauchy problem of the Laplace equation by Andrieux et al. in [11] and by Ben Belgacem et al. in $[12,13]$.

\section{The Cauchy-Steklov-Poincare Equation}

To keep the notational complexity to a minimum let us remove $\mathbf{x}$ from the notations. Let $\boldsymbol{\lambda}$ denote the unknown displacement vector on $\Gamma_{i}$. We consider both Dirichlet and Neumann elliptic problems obtained by duplicating the solution $u$ into a couple of vectors $\mathbf{u}_{\mathrm{N}}, \mathbf{u}_{\mathrm{D}}$. The Cauchy problem (6) is then split into:

$$
\left\{\begin{array} { c } 
{ - \operatorname { d i v } \sigma ( \mathbf { u } _ { \mathrm { N } } ( \mathrm { x } ) ) = f \quad \operatorname { i n } \Omega } \\
{ t = \sigma ( \mathbf { u } _ { \mathrm { N } } ( \mathrm { x } ) ) n = \tilde { \mathbf { t } } ( \mathrm { x } ) \operatorname { i n } \Gamma _ { \mathrm { c } } } \\
{ \mathbf { u } _ { \mathrm { N } } = \lambda \operatorname { i n } \Gamma _ { \mathrm { i } } }
\end{array} \left\{\begin{array}{c}
-\operatorname{div} \sigma\left(\mathbf{u}_{\mathrm{D}}(\mathrm{x})\right)=f \quad i n \Omega \\
\mathbf{u}_{\mathrm{D}}=\tilde{\mathbf{u}}(\mathrm{x}) \operatorname{in} \Gamma_{\mathrm{c}} \\
\mathbf{u}_{\mathrm{D}}=\lambda \operatorname{in} \Gamma_{\mathrm{i}}
\end{array}\right.\right.
$$
(1) for

If the pair $(\tilde{\mathbf{u}}, \tilde{\mathbf{t}})$ is compatible (i.e. a vectors field exists that verifies

Which $(\tilde{\mathbf{u}}, \tilde{\mathbf{t}})$ are the Cauchy data on $\Gamma_{c}$, the solution of the Cauchy problem (1-5) is recovered, i.e. $\mathbf{u}=\mathbf{u}_{\mathrm{D}}=\mathbf{u}_{\mathrm{N}}$ in $\Omega$, if and only if $\sigma\left(\mathbf{u}_{\mathrm{D}}(\lambda)\right) \mathbf{n}=\sigma\left(\mathbf{u}_{\mathrm{N}}(\lambda)\right) \mathbf{n}$ on $\Gamma_{\mathrm{i}}$

Now for $\boldsymbol{\mu}$, a displacements vector defined on $\Gamma_{i}$, the linear parts of $\mathbf{u}_{\mathrm{N}}(\boldsymbol{\mu})$ and $\mathbf{u}_{\mathrm{D}}(\boldsymbol{\mu})$ are denoted $\mathbf{u}_{\mathrm{N}}^{0}(\mu)$ and $\mathbf{u}_{\mathrm{D}}^{0}(\mu)$ which solve respectively: $\left\{\begin{array}{c}-\operatorname{div} \sigma\left(\mathbf{u}_{\mathrm{N}}^{0}(\mu)\right)=0 \quad \text { in } \Omega \\ t=\sigma\left(\boldsymbol{u}_{N}^{0}(\mu)\right) n=0 \text { in } \Gamma_{c} \\ \boldsymbol{u}_{N}^{0}(\mu)=\mu \text { in } \Gamma_{i}\end{array}\right.$

$$
\left\{\begin{array}{c}
-\operatorname{div} \sigma\left(\grave{\boldsymbol{u}}_{D}^{0}()\right)=f \quad \text { in } \Omega \\
\boldsymbol{u}_{D}^{0}(\mu) n=0 \quad \text { in } \Gamma_{c} \\
\boldsymbol{u}_{D}^{0}(\mu)=\mu \text { in } \Gamma_{i}
\end{array}\right.
$$

We consider also $\boldsymbol{u}_{N}^{*}$ and $\boldsymbol{u}_{D}^{*}$ such that:

$$
\left\{\begin{array} { c } 
{ - \operatorname { d i v } \sigma ( \boldsymbol { u } _ { N } ^ { * } ) = f \quad \text { in } \Omega } \\
{ t = \sigma ( \boldsymbol { u } _ { N } ^ { * } ) n = \tilde { \boldsymbol { t } } \text { in } \Gamma _ { c } } \\
{ \boldsymbol { u } _ { N } ^ { * } = 0 \text { in } \Gamma _ { i } }
\end{array} \left\{\begin{array}{c}
-\operatorname{div} \sigma\left(\boldsymbol{u}_{D}^{*}\right)=f \operatorname{in} \Omega \\
\boldsymbol{u}_{D}^{*}=0 \text { in } \Gamma_{c} \\
\boldsymbol{u}_{D}^{*}=0 \text { in } \Gamma_{i}
\end{array}\right.\right.
$$

And, by superposition, we obtain $\boldsymbol{u}_{N}(\mu)=\boldsymbol{u}_{N}^{0}(\mu)+\boldsymbol{u}_{N}^{*}$ and $\boldsymbol{u}_{D}(\mu)=\boldsymbol{u}_{D}^{0}(\mu)+\boldsymbol{u}_{D}^{*}$. With this partition, condition (8) is written as

$$
\sigma\left(\boldsymbol{u}_{D}^{0}(\lambda)\right) \boldsymbol{n}-\sigma\left(\boldsymbol{u}_{N}^{0}(\lambda)\right) \boldsymbol{n}=\sigma\left(\boldsymbol{u}_{N}^{*}\right) \boldsymbol{n}-\sigma\left(\boldsymbol{u}_{D}^{*}\right) \boldsymbol{n} \text { on } \Gamma_{\mathrm{i}}
$$

Using the following notations:

$S_{D}(\lambda)=\lambda\left(\lambda u^{0} \lambda_{D}(\lambda)\right) n, S_{N}(\lambda)=\sigma\left(u_{D}^{0}(\lambda)\right) n$ and $\chi=\left(\sigma\left(u_{N}^{*}\right)-\sigma\left(u_{D}^{*}\right)\right) n$

Equation (11) becomes: $S(\lambda)=S_{D}(\lambda)-S_{N}(\lambda)=\chi$ on $\Gamma_{i}$

Equation (12) is called the Steklov-Poincar'e interface equation and $S$ is the Steklov-Poincar'e operator. It is familiar in the domain decomposition framework [14] for the direct boundary value problem. More precisely, things happen as if vectors $u_{D}$ and $u_{N}$ were defined on two different domains with common boundary $\Gamma_{i}$. In this case, the equation (12) expresses the Neumann transmission condition, but the (-) sign in $S$ would be (+) in the domain decomposition formulation [14]. The (-) sign which appears in $S$ is at the origin of ill-posedness 
of the Cauchy problem. From the discrete point of view, the finite element discretization of $S$ leads to the Schur complement matrix [14]. It corresponds to having all interior nodes eliminated by static condensation [15]. In Ben Abdallah [16] a numerical study of the Shur complement matrix is performed for the Cauchy-Poisson problem. We will propose a study of the Cauchy problem in elasticity based on the Stecklov-Poincar'e equation in a forthcoming paper.

We now continue with the analogy with domain decomposition and show how the Cauchy-Steklov-Poincar'e equation can be expressed, as in domain decomposition, in terms of the Dirichlet-toNeumann problem.

\section{The Dirichlet-to-Neumann Solver for the Cauchy Problem}

When describing the Dirichlet-to-Neumann approach it should be noted that when the complete data are available on $\Gamma$, we have an overspecified boundary value problem $-\operatorname{div} \sigma(u)=f$ in $\Omega$

$$
\sigma(u) n=\tilde{t}, u=\tilde{u} \quad \text { on } \Gamma_{c} ; \sigma(u) n=\bar{t}, u=\bar{u} \quad \text { on } \Gamma_{i} ;
$$

This problem can be split into two well-posed subproblems with different boundary conditions. For one of them (Neumann/Dirichlet) conditions are Imposed on $\left(\Gamma_{c} / \Gamma_{i}\right)$

$$
\begin{aligned}
& -\operatorname{div} \sigma(\hat{u})=f \quad \text { in } \Omega \\
& \sigma(\hat{u}) n=\tilde{t} \quad \text { on } \Gamma_{c} \\
& \hat{u}=\bar{u} \quad \text { on } \Gamma_{i} \\
& -\operatorname{div} \sigma\left(\begin{array}{c}
i \\
u
\end{array}\right)=f \quad \text { in } \Omega \\
& \dot{u}=\tilde{u} \quad \text { on } \Gamma_{c} \\
& \sigma\left(\begin{array}{c}
i \\
u
\end{array}\right) n=\bar{t} \quad \text { on } \Gamma_{i} c
\end{aligned}
$$

Solving the Cauchy system (1)-(5) is achieved when extension $(\bar{t}, \bar{u})$ makes $\hat{u}$ and $\dot{u}$ coincide so the solution is then $u=\hat{u}=\dot{u}$.

Basically, the iterative method proposed for the Cauchy-Poisson problem and studied in Kozlov et al. [8], is derived from these observations: starting from an arbitrary prediction of the Dirichlet condition (here the displacement vector $\hat{u}$ ) on $\Gamma_{i}$, we add several corrections by solving alternately a Dirichlet on $\Gamma /$ Neumann on $\Gamma_{i}$ problem, where at each iteration the appropriate boundary data are inferred from the solution computed in the previous step. More specifically, we construct a sequence of a pair of vectors $\left(u_{N}^{(k)}, u_{D}^{(k)}\right)_{k}$ from the following recurrence: given $u_{D}^{(0)}$, the following systems are solved for each $: k \geq 0$

$$
\left\{\begin{array} { c } 
{ - \operatorname { d i v } \sigma ( u _ { N } ^ { ( k + 1 ) } ) = f \quad \text { in } \Omega } \\
{ \sigma ( u _ { N } ^ { ( k + 1 ) } ) n = \tilde { t } \text { on } \Gamma _ { c } } \\
{ u _ { N } ^ { ( k + 1 ) } = u _ { D } ^ { ( k ) } \text { on } \Gamma _ { i } }
\end{array} \left\{\begin{array}{c}
-\operatorname{div} \sigma\left(u_{D}^{(k+1)}\right)=f \quad \text { in } \Omega \\
u_{D}^{(k+1)}=\tilde{u} \text { on } \Gamma_{c} \\
\sigma\left(u_{D}^{(k+1)}\right) n=\sigma\left(u_{N}^{(k+1)}\right) n \text { on } \Gamma_{i}
\end{array}\right.\right.
$$

The convergence of the alternating method toward the solution of the Cauchy problem and its stabilizing properties are established by Kozlov et al. [8] for the steady state thermal case. In the linear elastic framework, no convergence result has been proved till now but the result of convergence established by Koslov et al. may be applied for any elliptic operators. When convergence is achieved, we may obtain $(\bar{t}, \bar{u})=(\sigma(u) n, u)$ on $\Gamma_{i}$. By using straightforward computations, it can be established that the Dirichlet-to-Neumann scheme can be interpreted as a preconditioned Richardson procedure for the CauchySteklov-Poincaré equation. For this purpose, the Dirichletto-Neumann algorithm is rewritten, using the previous notations, as follows: Given $\lambda^{0}$,

$$
\left\{\begin{array} { c } 
{ - \operatorname { d i v } \sigma ( u _ { N } ^ { ( k + 1 ) } ) = f \quad \text { in } \Omega } \\
{ u _ { N } ^ { ( k + 1 ) } = \tilde { u } \text { on } \Gamma _ { c } } \\
{ u _ { N } ^ { ( k + 1 ) } = \lambda ^ { ( k ) } \text { on } \Gamma _ { i } }
\end{array} \left\{\begin{array}{c}
-\operatorname{div} \sigma\left(u_{D}^{(k+1)}\right)=f \quad \text { in } \Omega \\
\sigma\left(\mathrm{u}_{\mathrm{D}}^{(\mathrm{k}+1)}\right) n=\tilde{\mathrm{t}} \quad \text { on } \Gamma_{\mathrm{c}} \\
\sigma\left(\mathrm{u}_{\mathrm{D}}^{(\mathrm{k}+1)}\right) n=\sigma\left(\mathrm{u}_{\mathrm{N}}^{(\mathrm{k}+1)}\right) n \text { on } \Gamma_{\mathrm{i}}
\end{array}\right.\right.
$$

The last equality $\sigma\left(\mathrm{u}_{\mathrm{D}}^{(\mathrm{k}+1)}\right) \mathrm{n}=\sigma\left(\mathrm{u}_{\mathrm{N}}^{(\mathrm{k}+1)}\right) \mathrm{n}$ on $\Gamma_{\mathrm{i}}$, can be written as

$$
\sigma\left(\mathrm{u}_{\mathrm{D}}^{0(\mathrm{k}+1)}\right) \mathrm{n}+\sigma\left(\mathrm{u}_{\mathrm{D}}^{*}\right) \mathrm{n}=\sigma\left(\mathrm{u}_{\mathrm{N}}^{0(\mathrm{k}+1)}\right) \mathrm{n}+\sigma\left(\mathrm{u}_{\mathrm{N}}^{*}\right) \mathrm{n} \cdot
$$

Since $\sigma\left(\mathrm{u}_{\mathrm{D}}^{0(\mathrm{k}+1)}\right) \mathrm{n}=\mathrm{S}_{\mathrm{D}} \lambda^{(\mathrm{k}+1)}$ and $\sigma\left(\mathrm{u}_{\mathrm{N}}^{0(\mathrm{k}+1)}\right) \mathrm{n}=\mathrm{S}_{\mathrm{N}} \lambda^{(\mathrm{k})}$ on $\Gamma_{\mathrm{i}}$ it follows that $\mathrm{S}_{\mathrm{D}} \lambda^{(\mathrm{k}+1)}=\mathrm{S}_{\mathrm{N}} \lambda^{(\mathrm{k})}+\chi$, and therefore $\lambda^{(\mathrm{k}+1)}=\lambda^{(\mathrm{k})}-\mathrm{S}_{\mathrm{D}}^{-1}\left(\mathrm{~S} \lambda^{\mathrm{k}}-\chi\right)$ We are thus left with a Richardson procedure for the Cauchy-Steklov-Poincaré equation (12) with the operator $S_{\mathrm{D}}$ as a preconditioner. In the following section we will discuss the efficiency of the Dirichlet-to-Neumann algorithm presented above as a numerical solver for several particular Cauchy problems in linear elasticity.

\section{Applications}

This section is devoted to the presented method in two situations taken from engineering mechanics. The first example concerns contact identification on an inaccessible contact area. The second deals with coating defect identification.

\section{Contact pressures identification}

Domain $\Omega$ is a square plate $\left(1 .{ }^{\star} 1\right.$.) with a circular hole $(\mathrm{R}=0.20225)$, where a fixed rigid disc $\mathrm{R}=0.2$ is placed. Figure 1 shows the geometry and boundary conditions applied to the plate. The mechanical properties of the plate are given in Table 1 . When tractions are applied on the plate, it comes into contact with the lower part of the disc (Figure 1).
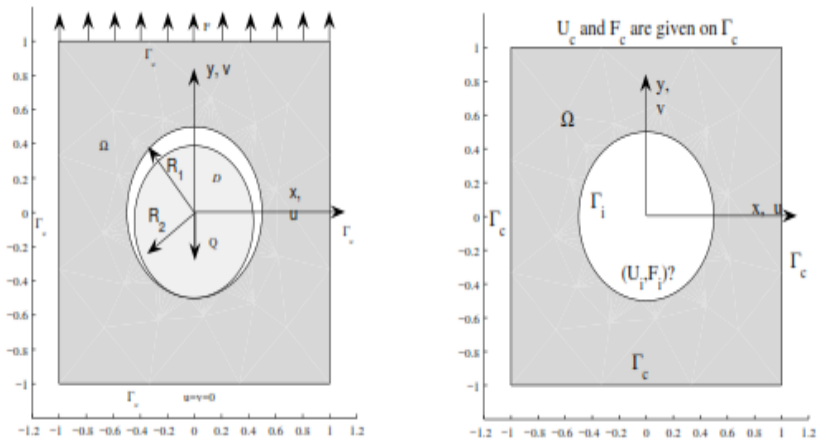

Figure 1: Geometry of the problem studied 


\begin{tabular}{|l|c|}
\hline Disc and plate & Aluminium \\
\hline Modulus of elasticity & $E=70000 \mathrm{MPA}$ \\
\hline Poison coefficient & $u=0.31$ \\
\hline Friction coefficient & $\mu=0$ \\
\hline Load applied to the plate & $\mathrm{F}=1^{+7} \mathrm{~N} / \mathrm{m}$ \\
\hline
\end{tabular}

Table 1: Mechanical characteristic of the plate and the disc.

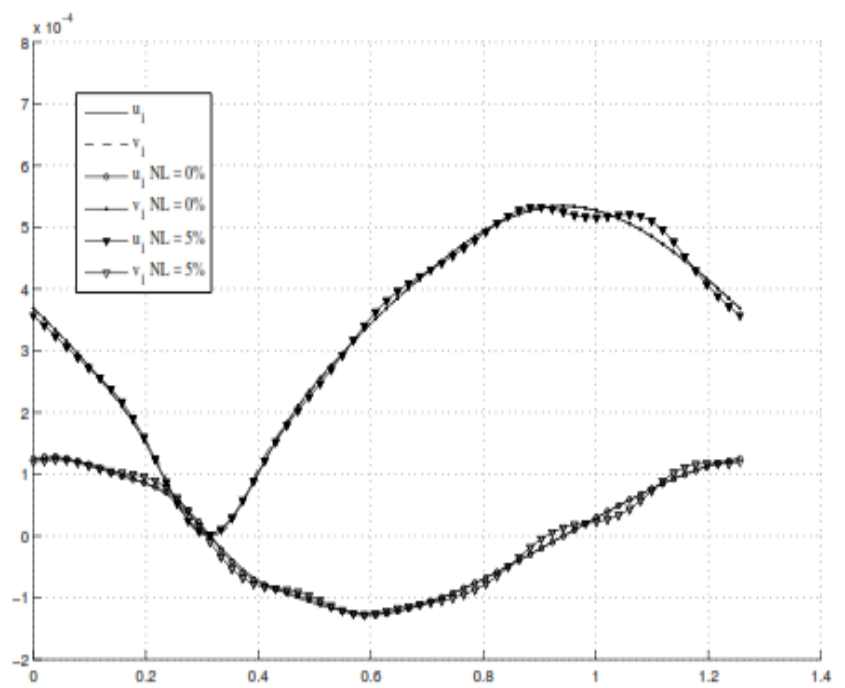

Figure 2: Reconstruction of horizontal $(\mathrm{U})$ and vertical $(\mathrm{V})$ displacements on the internal boundary of the plate for noise free $(\mathrm{N} . \mathrm{L} .=0 \%)$ and noisy (N. $\mathrm{L} .=5 \%)$ data.

The problem is to identify the contact pressure distribution and the displacements on the interface between the plate and the disc, by using overs pecified data provided for the external boundary. These overs pecified data are generated by solving a direct problem using Hertz's analytical contact law. Here, we consider a frictionless contact so that only normal pressure is taken in account. Moreover, plane strain hypothesis is assumed.

The results obtained by solving the corresponding Cauchy problem are the normal stress components and the displacements field on $\Gamma_{i}$. Hence, the contact zone is the part of the boundary where the normal stress components are not null.

When carrying out an identification based on measurements, it must be kept in mind that measured data are subject to noise whose effects have to be studied. In this case, the data are synthetic, and therefore suffer from some errors (approximation error, roundoff error, ... etc). We added a noise generated by a MATLAB routine (randn) to the computational noise. The displacement measurements are polluted by a noise level at $5 \%$. Figure 2 depicts the horizontal (resp. vertical) displacements $U$ (resp.) reconstruction on the internal boundary of the plate. Figure 3 shows the identification of the normal stress distribution on the internal boundary of the plate. As expected, displacements reconstruction is better than that for the stresses, particularly when the data are noisy. The reason is that the stresses are homogeneous with the displacements gradient and it is well known that the derivation is an ill-posed operation (the influence of noise is considerable). The identification is very satisfactory free noisy data. For noise-free data.

For noisy data, the contact zone is well localized and the contact pressures are recovered correctly. However, some fairly significant oscillations appear on the free boundary.

\section{Coating defect identification}

The identification of inter-facial cracks is a crucial issue in detecting coating defects or delamination in composite material. Our second experiment focuses on the detection of curved inter-facial cracks. We consider a double-layered annular domain centered at the origin with an inner-radius $0.6\left(\Gamma_{c}\right)$, middleradius $0.8\left(\Gamma_{i}\right)$ and an outer-radius $1\left(\Gamma_{c t}\right)$ as shown on Figure 4 . The coating defect lies at $\Gamma_{i}$. The simulation is run using synthetic data generated by a finite element resolution of the direct problem. The direct problem is solved with prescribed displacements on the inner boundary and with prescribed surface tractions acting on the outer boundary. The cracks are approximated by two thin cavities on which a homogeneous Neumann condition is prescribed. In order to detect the coating defect, two Cauchy problems are solved. The first, $\mathrm{P}_{+}$, is defined on subdomain $\Omega$ where the overspecified data are given on the external boundary $\Gamma_{c+}$ and the unknowns are identified on the boundary $\Gamma_{\mathrm{i}}$. The second Cauchy problem $\mathrm{P}_{-}$is defined on subdomain

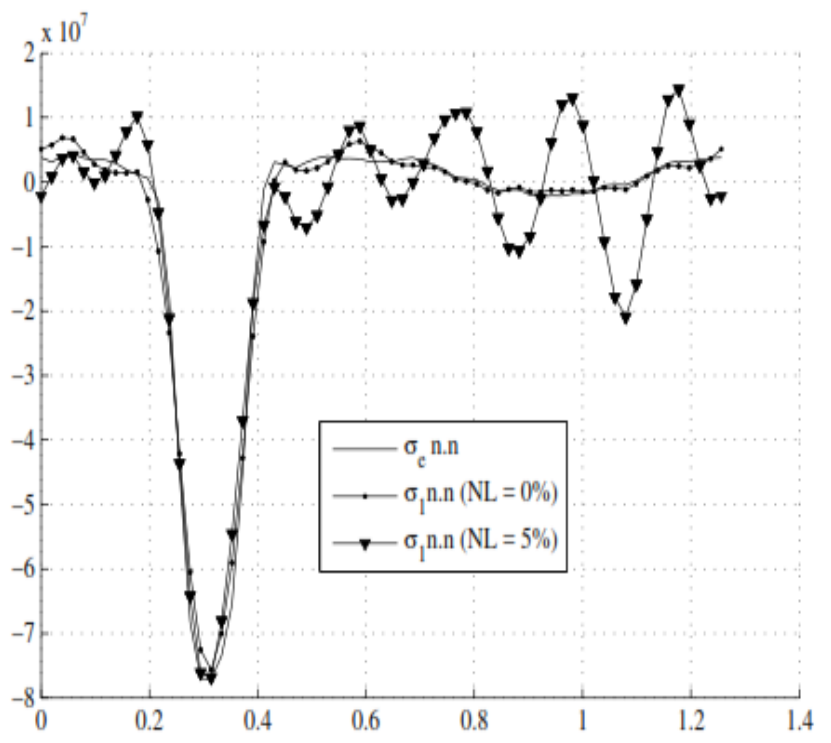

Figure 3: Reconstruction of the normal stress distribution on the internal boundary of the platefor noise free (N.L. $=0 \%$ ) and noisy (N.L. $=5 \%)$ data.

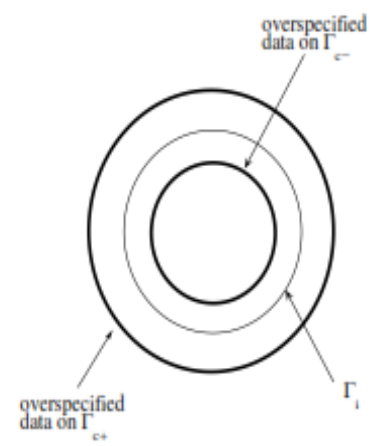

Figure 4: Geometry of the domain with a coating defect. 

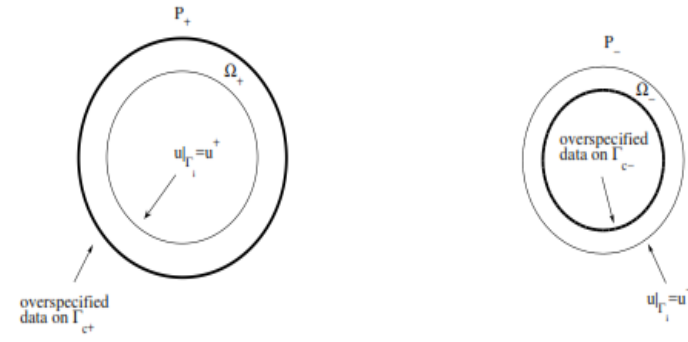

Figure 5: Geometries of subdomains on which Cauchy problems are solved.

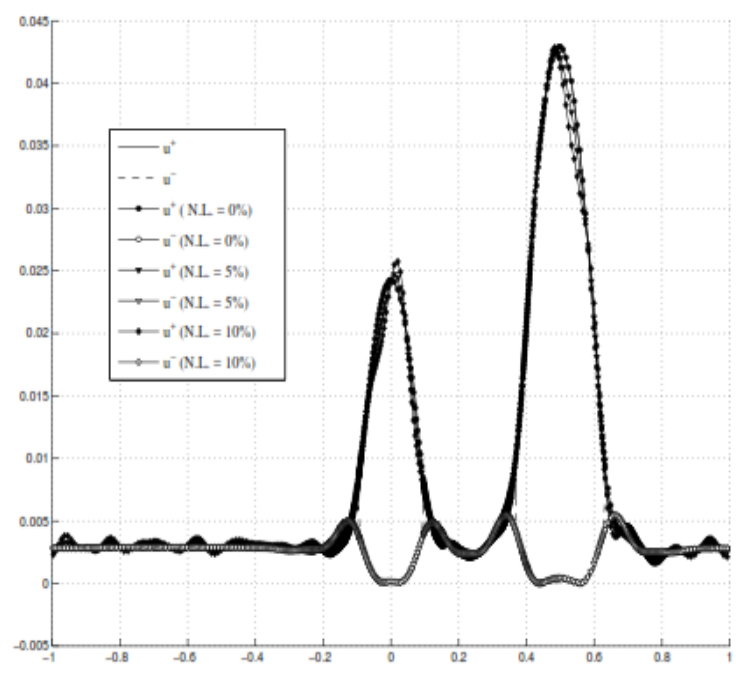

Figure 6: Interfacial displacement identification.

$\Omega_{-}$where the overspecified data are given on internal boundary $\Gamma_{c}$ and the unknowns are identified on boundary (Figure 5). Among the unknowns we are only interested in the displacements. In fact, if we use $\mathrm{u}^{+}$(resp. $\mathrm{u}^{-}$) to denote the displacements field on $\Gamma_{\mathrm{i}}$ provided by $\mathrm{P}_{+}$ (resp.P.), the cracks will appear as the part of $\Gamma_{\mathrm{i}}$ where the jump of the displacements vector $\left[\mathrm{u}^{+}-\mathrm{u}^{-}\right]$does not vanish.

Two interfacial cracks with different widths are simulated. In this case also we tested the reconstruction algorithm in the case of noise free and noisy data. The displacements were polluted with noise at $5 \%$ and $10 \%$ level. The reconstructed $\mathrm{u}^{-}$and $\mathrm{u}^{+}$and the reconstruction of the jump $\left[\mathrm{u}^{+}-\mathrm{u}^{-}\right]$across the interface are plotted in Figures 6 and 7. It can be seen again that good agreement is achieved with the exact solution, even for noisy cases. It seems that the width of the crack has no influence on the accuracy of the reconstructed procedure: both cracks are well recovered.

\section{Conclusion}

In this work we presented a numerical method for solving the Cauchy problem in the framework of linear elasticity. The method proposed was applied in two practical situations taken from engineering mechanics: contact pressure recovery and coating defect identification. We also presented an alternative formulation of the Cauchy problem which lead to an operator acting on the boundary of the unknown: the

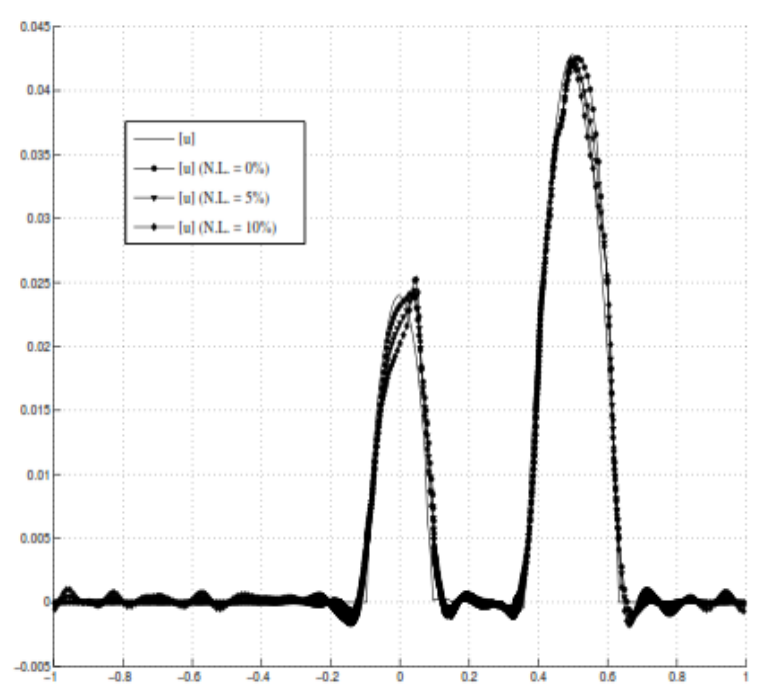

Figure 7: Interfacial jamp displacement identification.

Steklov-Poincaré operator. The study of the properties of this operator for Neumann and Dirichlet variable, comparison with the energy approach recently presented by Baranger et al. $[17,18]$ and its use in practical situations will be subject of a forthcoming paper [19-22].

\section{Acknowledgment}

This work was funded by the Ministère de la Recherche Scientifique, de la Technologie et du Développement des Compétences (MRSTDC, Tunisia) within the LAB-STI-02 program.

\section{References}

1. Hadamard J (1953) Lectures on Cauchy's problem in linear partial differential equation, Dover

2. Yeih WC, Koya T, Mura T (1993) An inverse problem in elasticity with partially overspecified boundary conditions, Theoretical approach, Transactions. ASME Journal of Applied Mechanics 60: 595-600.

3. Koya T, Yeih WC, Mura T (1993) An inverse problem in elasticity with partially over specified boundary conditions. II. Numerical details. Transactions ASME Journal of Applied Mechanics 60: 601-606.

4. Marin L, Elliot L, Ingham DB, Lesnic D (2001) Boundary element method for the Cauchy problem in linear elasticity. Engineering Analysis with Boundary Elements 25(9): 783-793.

5. Marin L, Lesnic D (2002) Boundary element solution for the Cauchy problem in linear elasticity using singular value decomposition. Comput Methods Appl Mech Engrg, 191: 3257-3270.

6. Huang $\mathrm{CH}$, Shih WY (1999) An inverse problem in estimating interfacial crack in bimaterials by boundary element technique. International Journal for Numerical Methods in Engineering 45: 1547-1567.

7. Weikl W, Andra H, Schnack E (2001) An alternating iterative algorithm for the reconstruction of internal cracks in 3D solid body. Inverse Problems 17: 19571975.

8. Koslov VA, Maz'ya VG, Fomin AV (1991) An iterative method for solving the Cauchy problem for elliptic equations. Comput Meth Math Phys 31: 45-52.

9. Riadh Ben Fatma, Mejdi Azaez, Amel Ben Abda, Nabil Gmati (2007) a Missing boundary data recovering for the Helmholtz problem. Comptes Rendus M'ecanique 335: 787-792.

10. Mohamed Larbi Kadri, Jalel Ben Abdallah, Thouraya Nouri Baranger (2011) Identification of internal cracks in a three-dimensional solid body via Steklov Poincar approaches. Comptes Rendus M'ecanique 339: 674-681. 
Citation: Kadri ML (2015) Contact Pressures and Cracks Identification by using the Dirichlet-to-Neumann Solver in Elasticity. J Appl Mech Eng 4: 152. doi:10.4172/2168-9873.1000152

11. Andrieux S, Baranger TN, Ben Abda A (2006) Solving Cauchy problems by minimizing an energy-like functional. Inverse Problem 22: 115-133.

12. Ben Belgacem F, El Fekih (2006) On Cauchy's problem: I A variational Stecklov-Poincar'e theory. Inverse Problem 21: 1915-1936.

13. Azaiez M, Ben Belgacem F, El Fekih (2005) On Cauchy's problem: II Completion, regularization and approximation. Inverse Problem 22: 1307-1336.

14. Quarteroni A, Valli A (1999) Domain Decomposition Methods for Partial Differential Equations. Oxford University Press.

15. Przemieniecki JS (1985) Theory of Matrix Structural Analysis, Dover: New York.

16. Ben Abdallah J (2007) A conjugate gradient type method for the Stecklov Poincar'e formulation of the Cauchy-Poisson Problem. International Journal of Applied Mathematics and Mechanics 3: 27-40.
17. Baranger TN, Andrieux S (2006) An energy approach to solve a Cauchy problem in elasticity, III European Conference on Computational Mechanics: Solids, Structures and Coupled Problems in Engineering, ECCM-2006, Lisbon, Portugal, 5-8 June, 2006.

18. Baranger TN, Andrieux S (2007) An optimization approach to solve Cauchy problem in linear elasticity. Journal of Structural and Multidisciplinary Optimization to appear.

19. Andrieux S, Ben Abda A, Baranger TN (2005) Data completion via an energy like error functional. Comptes Rendus M'ecanique 333: 171-177.

20. Azaiez M, Ben Abda A, Ben Abdallah J (2005) Revisiting the Dirichlet-to Neumann solver for data completion and application to some inverse problems. International Journal of Applied Mathematics and Mechanics 1: 106-121.

21. Johnson KL (1985) Contact mechanics. Cambridge: Cambridge, University Press.

22. Tikhonov AN, Arsenin VY (1977) Solution to III-posed Problems, Winston Wiley 